\title{
Early maladaptive schemas and harm avoidance as mediating factors between early life stress and psychiatric symptoms in adults
}

\author{
Ismael F. da Costa, ${ }^{1}$ (D) Maria P. Tomaz, ${ }^{1}$ iD Giselle do N. Pessoa, ${ }^{1}$ iD Hortência de S. Miranda, ${ }^{2}$ iD \\ Melyssa K. Galdino ${ }^{1}$ iD \\ ${ }^{1}$ Universidade Federal da Paraíba (UFPB), João Pessoa, PB, Brazil. ${ }^{2}$ Centro Universitário de João Pessoa (UNIPÊ), João Pessoa, PB, Brazil.
}

\begin{abstract}
Objective: This study investigated how factors of temperament and early maladaptive schemas predict psychiatric symptoms, as well as how they mediate the relation between early life stress and psychiatric symptoms in adults.

Methods: A cross-sectional study was conducted with a sample of 200 university students. Data was collected through a sociodemographic questionnaire, the Adult Self-Report Inventory, the Childhood Trauma Questionnaire, the Young Schema Questionnaire, and the Temperament and Character Inventory-Revised.

Results: A model including early maladaptive schemas, harm avoidance (temperament factor), and early life stress explained $69 \%$ of the variation of the psychiatric symptoms; among the predictors, early maladaptive schemas explained $31 \%$ of psychiatric symptoms, while harm avoidance explained $25 \%$. Most of the predictive power associated with early life stress can be better explained by early maladaptive schemas and, to a lesser extent, harm avoidance.

Conclusion: By managing these processes therapeutically, deleterious effects associated with early life stress can be minimized.
\end{abstract}

Keywords: Early life stress; temperament; personality; psychiatric symptoms

\section{Introduction}

In recent years, fruitful and enlightening research has been conducted on the relation between early life stress (ELS) or childhood trauma and the development of mental disorders in adulthood. ${ }^{1}$ Studies have shown the impact that childhood adversity can have on the development of mental disorders. ${ }^{2}$ Although there are several forms of ELS, some have been more investigated than others due to their high correlation with psychiatric disorders. ${ }^{3}$ A close association has been found between physical, sexual, and emotional abuse, as well as emotional and physical neglect, with various mental disorders. ${ }^{4,5}$

This association has been linked with the effects of stress on the brain during its development in childhood and adolescence. It has been argued that ELS can permanently change functional patterns in the hypothalamic-pituitary-adrenal (HPA) axis, which is responsible for regulating neuroendocrine mechanisms related to stress. ${ }^{6}$ These changes lead to an increased release of glucocorticoid and mineralocorticoids, which affect the development of brain structures such as the amygdala, hippocampus, and prefrontal cortex. The receptors for

Correspondence: Ismael Ferreira da Costa, Universidade Federal da Paraíba, Campus I, Conjunto Castelo Branco I, CEP 58051-900, João Pessoa, PB, Brazil.

E-mail: ismaelferr@gmail.com

Submitted Jul 03 2019, accepted Jan 10 2020, Epub May 112020. these hormones emit negative feedback, which regulates HPA functioning. ${ }^{7}$ Thus, maladaptive functioning of the HPA axis and its effects on the development of brain structures could mediate between ELS in childhood and adolescence and the emergence of mental disorders in adulthood, an explanatory model that is widely accepted today and is supported by many studies. ${ }^{8}$

However, despite the importance of mediating factors in neuroendocrine function, little emphasis has been given to other mediating factors, such as personality traits, which can develop as a direct or indirect result of ELS.

Cloninger's Psychobiological Model $^{9}$ postulates that personality consists of temperament and character, two interrelated areas that interact dynamically and are involved in the development of an individual's psychological functioning, leading to the establishment of personality. Temperament is predominantly a hereditary field and includes traits that manifest themselves in early childhood and are reasonably independent from the external environment. It involves automatic responses to emotionally relevant stimuli, including basic emotional responses, such as fear, anger, and attachments.
How to cite this article: Costa IF, Tomaz MP, Pessoa GN, Miranda HS, Galdino MK. Early maladaptive schemas and harm avoidance as mediating factors between early life stress and psychiatric symptoms in adults. Braz J Psychiatry. 2020;42:489-495. http://dx. doi.org/10.1590/1516-4446-2019-0593 
Character, on the other hand, involves individual differences in higher cognitive processes that underlie the goals and values a person develops. Unlike temperament, character is more flexible and can change as time goes by, being influenced by factors such as temperament traits, sociocultural pressure, and an individual's unique life experiences. ${ }^{10}$

The development of the personality is a complex adaptive effort towards self-organization, aiming at an appropriate adjustment between internal needs (temperament) and external demands (environmental experiences, norms, and social customs). ${ }^{9}$ This adaptation can occur in a dysfunctional manner either due to previous and inflexible behavioral provisions, a pathogenic environment, or both. Therefore, depending on how they are constructed, temperament factors can act as a diathesis, leading to increased susceptibility to the development of mental disorders, especially when associated with ELS. Several studies have indicated that the temperament factor harm avoidance (HA) is closely associated with the development of various mental disorders. ${ }^{11}$

Young, Klosko \& Weishaar developed a cognitive model called schema therapy, which, although initially restricted to a clinical focus, is one of the most important current cognitive models of personality, as well as one of the broadest and most integrative ones, since it involves concepts from several theories. One of the main assumptions of schema therapy is that the traumatic childhood events play a decisive role in the development of dysfunctional cognitive structures, called early maladaptive schemas (EMS), which are defined as broad and diffuse cognitive patterns consisting of memories, emotions, and bodily sensations that are acquired in early development and produced throughout life. The authors of this model propose that EMS established during childhood are stored in a long-term memory system and gradually acquire a format that consists of several pieces of information that are automatically activated in the presence of stressors, particulary those that involve aspects of the event that caused the original EMS, and lead emotional instability. Although the authors do not attribute these dysfunctional schemas exclusively to harmful experiences in childhood or adolescence, they state that schemas that are formed earlier and are related to traumatic events tend to be more invasive and have a greater impact on information processing and, thus, are more harmful. ${ }^{12}$

Studies from several countries have used the the Young Schema Questionnaire (YSQ) in clinical and nonclinical populations. This instrument was designed to detect EMS and has identified, until now, 18 EMS that are central to human cognition. These EMS are related to specific themes and are developed under adverse conditions of abuse and neglect. ${ }^{13} \mathrm{~A}$ number of studies have pointed out the close relationship between EMS and mental disorders. ${ }^{14}$

Various studies have investigated the relationship between childhood trauma and personality, finding that trauma is associated with reduced expression of adaptative personality traits and increased expression of potentially dysfunctional ones. ${ }^{15}$ Although only a few of these studies have included temperament and character, they found that ELS is associated with lower expression of self directedness and cooperativity, which are important predictors of emotional adjustment, and that temperament factors are not related to ELS. ${ }^{16}$ Several studies have found a close association between ELS and EMS, ${ }^{17}$ including a relationship between EMS and various forms of abuse and neglect. Some have suggested that EMS is an effective mediator for mental disorders. ${ }^{18}$

Although the association between childhood trauma, personality, and mental disorders has been generally well documented in the literature, it is still unclear how personality factors (such as temperament) and EMS mediate the relationship between ELS and psychiatric symptoms. Thus, this study aimed to investigate how the HA temperament factor and EMS mediate the relationship between ELS and psychiatric symptoms in a non-clinical sample. It was hypothesized that these factors significantly mediate the well-documented harmful effects of ELS on the development of psychiatric symptoms.

\section{Methods}

\section{Participants}

A non-probability sampling process (snowball sampling or chain-referral sampling) resulted in 236 participants, of whom 36 were excluded from the final sample for not correctly filling out all the instruments. Thus, the final sample, which was predominantly female $(n=170,65 \%)$, included 200 participants, all of whom were university students between 18 and 30 years old (mean $22.70 \pm 3.52$ ).

\section{Instruments}

\section{Sociodemographic questionnaire}

The questionnaire consisted of 15 general information items about the participants, including age, gender, religion, marital status, education level, employment situation, family income, and family history of mental disorders.

\section{Early life stress}

Childhood trauma was investigated through the Childhood Trauma Questionnaire ${ }^{19}$ (CTQ), a retrospective interview with 28 Likert-scale items ranging from 0 (never) to 5 (very often) that evaluate five subtypes of childhood trauma: physical, emotional, and sexual abuse, and physical and emotional neglect. The scores range from 5 to 25 for each subtype. Individuals with moderate to severe or severe to extreme scores in any trauma subtype are identified as having a history of ELS. Respondents who score none to minimum or light to moderate are not considered to have such a history. The total score of the five trauma subtypes was also used in this study. In this study's sample, the CTQ presented a high confidence level (Cronbach's alpha $=0.89$ ).

\section{Psychiatric symptoms}

Psychiatric symptoms were assessed through the Adult Self-Report Inventory ${ }^{20}$ (ASRI), which consists of 
136 Likert-scale items ranging from 0 (never) to 3 (very often) that quantitatively evaluate psychiatric symptoms from 19 mental disorders included in the DSM-IV. The instrument produces two scores: cut-off values for each disorder that indicate the presence or not of symptoms, and a total score for all disorders, which was used in this study's analysis. The ASRI presented a high confidence level (Cronbach's alpha $=0.87$ ).

\section{Cognitive schemas}

Maladaptive shemas were assessed with the YSQ: Short Form $3,{ }^{12}$ which evaluates 18 EMS through 90 Likert-scale items ranging from 1 (completely false) to 6 (describes me perfectly). The questionnaire includes individual scores for each schema and a total score for all 18 schemas, which was used in this study's analysis. In this study's sample, the YSQ: Short Form 3 presented a high confidence level (Cronbach's alpha $=0.92$ ).

\section{Temperament and character}

Temperament and character were assessed with the Temperament and Character Inventory-Revised ${ }^{9}(\mathrm{TCl}-\mathrm{R})$, which consists of 240 Likert-scale items ranging from 1 (definitely false) to 5 (definitely true). There are total and mean scores for four temperament factors and three character factors. In this study, only the total HA temperament factor score was used. In this study's sample, the TCl-R presented a high confidence level (Cronbach's alpha $=0.87$ ).

\section{Procedures}

Data was collected from one participant at a time. To avoid fatigue from the extensive nature of the protocol, data was collected in two sessions. First, after providing written informed consent, the participants responded to the sociodemographic questionnaire, the ASRI, and the YSQ: Short Form 3, which took a total of approximately 50 minutes. The second session included the CTQ and the TCl-R, which took approximately 40 minutes. Clear instructions were provided prior to administering the instruments. The two data collection sessions occurred a maximum of five days apart.

\section{Data analysis}

The data were analyzed in SPSS version 22.0. Pearson's test was used for the correlation analysis. From the most significant correlations, a stepwise multiple regression was performed.

The PROCESS ${ }^{21}$ tool was used to confirm mediating effects through a bootstrap confidence interval test with 5,000 resamplings. ${ }^{22}$ For all analyzed data, differences with $p<0.05$ were considered significant.

\section{Ethics statement}

Prior to data collection, this study was approved by the research ethics committee of the Universidade Federal da
Paraíba, Centro de Ciências da Saúde (protocol 0141/15; CAEE: 43221215.6.00005188). The study was conducted according to Resolution 466/12 of the Brazilian National Health Council, which oversees research involving human beings.

\section{Results}

\section{Descriptive statistics}

Regarding childhood trauma, the participants' mean total CTQ score was $41.02(S D=11.83)$. Among the stress subtypes, emotional neglect (mean $[\mathrm{M}]=10.38, \mathrm{SD}=$ 4.14) and emotional abuse $(M=10.36, S D=4.38)$ had the highest mean scores, while sexual abuse had the lowest mean score $(M=6.19, S D=3.06)$. The total mean score obtained for psychiatric symptoms was 35.52 (SD = 1,41). Concerning maladaptive schemas, the total mean YSQ score was 173.76 (SD = 57.99). Finally, on the TCl$\mathrm{R}$, the mean HA score was $103.22(\mathrm{SD}=17.18)$. $\mathrm{HA}$ was the final dimension analyzed due to its intimate relationship with mental disorders. Significant correlations were found between ELS and psychiatric symptoms $(r=0.46$, $p<0.01)$, ELS and EMS $(r=0.43, p<0.001)$, and ELS and $\mathrm{HA}(r=0.20, p<0.01)$. A correlation matrix with all the variables is shown in Table 1.

\section{Hierarchical multiple regression}

Based on the correlation results, a hierarchical multiple regression was performed with ELS (total score), EMS (total score), and HA (total score), which were assumed to be predictor variables for psychiatric symptoms (total score), the outcome variable. The three selected predictor variables significantly explained the occurrence of psychiatric symptoms: EMS 31\%, HA 25\%, and ELS 14\%. EMS had the greatest influence on psychiatric symptoms $\left(R^{2}=0.31, F_{2,161}=121.26, p<0.001\right)$, followed by $H A$ $\left(R^{2}=0.25, F_{1,162}=54.78, p<0.001\right)$, and ELS $\left(R^{2}=\right.$ $\left.0.14, F_{2,163}=49.933, p<0.001\right)$. Together, these three variables predicted $69 \%\left(R^{2}=0.69, F_{3,161}=160.76, p<\right.$ 0.001 ) of the psychiatric symptoms.

Table 2 shows the coefficients obtained for predictor variables in the three-step regression analysis. In the first block, HA had a coefficient of $\beta 0.51(t=7.40, p<0.001)$. When ELS $(\beta=0.36, t=5.98, p<0.001)$ was added in block 2, the HA coefficient reduced from 0.51 to 0.44 $(t=6.81, p<0.001)$, which suggests a small interaction between the two variables, although both still continued to significantly predict psychiatric symptoms. In block 3 ,

Table 1 Correlation between early life stress, psychiatric symptoms, early maladaptive schemas and harm avoidance

\begin{tabular}{|c|c|c|c|c|}
\hline Variables & 1 & 2 & 3 & 4 \\
\hline 1. Early life stress & 1.00 & & & \\
\hline 2. Psychiatric symptoms & $0.46 *$ & 1.00 & & \\
\hline 3. Early maladaptive schemas & $0.43^{\dagger}$ & $0.81^{\dagger}$ & 1.00 & \\
\hline $\begin{array}{l}\text { 4. Harm avoidance (temperament } \\
\text { factor) }\end{array}$ & $0.20 *$ & $0.49^{\dagger}$ & $0.44^{\dagger}$ & 1.00 \\
\hline
\end{tabular}

$* p<0.01 ;{ }^{\dagger} p<0.001$. 
including EMS $(\beta=0.66, t=12.67, p<0.001)$ led to a clear reduction in the ELS $(\beta=0.15, t=3.35, p<0.01)$ and HA coefficients $(\beta=0.18, t=3.59, p<0.001)$. In this case, there was a significant interaction between $\mathrm{HA}$ and ELS. Including EMS led to a reduced expression of ELS, whose coefficient was lower than in block two $(\beta=0.36$, $\mathrm{t}=3.26, \mathrm{p}<0.01)$. Part of the predictive power of ELS is mediated by EMS; although it is a significant predictor of psychiatric symptoms, the symptoms are better explained in conjunction with EMS. EMS had a similar effect on

Table 2 Coefficients of the hierarchical multiple regression analysis for psychiatric symptoms

\begin{tabular}{lccc}
\hline \multirow{2}{*}{ Models } & \multicolumn{3}{c}{ Psychiatric symptoms } \\
\cline { 2 - 4 } & $\mathrm{B}$ & SE B & $\beta$ \\
\hline Constant & -24.89 & 8.07 & \\
1 (HA) & 0.96 & 0.12 & $0.51^{*}$ \\
& & & \\
Constant & -39.80 & 7.83 & $0.44^{*}$ \\
2 (HA) & 0.81 & 0.11 & $0.36^{*}$ \\
(ELS) & 0.57 & 0.10 & \\
Constant & -34.94 & 5.55 & $0.18^{*}$ \\
3 (HA) & 0.35 & 0.09 & $0.15^{\dagger}$ \\
(ELS) & 0.23 & 0.76 & $0.66^{*}$ \\
(EMS) & 0.22 & 0.18 &
\end{tabular}

$\mathrm{B}=$ unstandardized coefficient (beta); $\beta=$ standardized coefficient (beta); ELS = early life stress; EMS = early maladaptive schemas; $\mathrm{HA}=$ harm avoidance; SE = standard error.

$* p<0.001 ;{ }^{\dagger} p<0.01$.

Table 3 Mediation model for early life stress (predictor variable), harm avoidance (mediator variable), and psychiatric symptoms (output or result variable)

\begin{tabular}{lc}
\hline Effects & $\mathrm{R}$ \\
\hline Total (c) & $0.73^{*}$ \\
Direct (c') & $0.60^{*}$ \\
Indirect (a.b) & $0.13^{*}$ \\
Indirect/total (a.b/c) & $0.18^{*}$ \\
\hline
\end{tabular}

$a=$ effect of early life stress on harm avoidance; $b=$ effect of harm avoidande on psychiatric symptoms; $c=$ total effect of early life stress on psychiatric symptoms; $c^{\prime}$ = direct effect of early life stress on psychiatric symptoms.

Bootstrapping $=5,000$ resamples $(95 \%$ confidence interval). $* p<0.001$.
HA ( $\beta=0.18, t=3.59, p<0.001)$, significantly reducing its coefficient in block 3 . In this case, the association between HA and psychiatric symptoms is mediated by EMS and is better explained in conjunction with it.

Two interactions observed in the regression led to two mediation models. In both, ELS was a predictor variable and psychiatric symptoms were the output variable. HA was the mediating variable in the first model (Table 3 and Figure 1), while EMS was the mediating variable in the second model (Table 4 and Figure 2).

The data presented in Table 3 and Figure 1 clarify the interaction between HA and ELS. The direct effect ( $c^{\prime}$ ) $(r=0.60, p<0.001,95 \% \mathrm{Cl} 0.4-0.7)$ between ELS and psychiatric symptoms represents a significant part of the total effect (c) $(r=0.73, p<0.01,95 \% \mathrm{Cl} 0.5-0.9)$, which is indicative of a subtle mediation by HA. The indirect effect (a.b) observed is small $(R=0.13, p<0.001,95 \%$ $\mathrm{Cl}$ 0.01-0.2), which represents $18 \%$ of the total effect, compared with the $82 \%$ allocated to the direct effect of the ELS on psychiatric symptoms. According to these data, HA and ELS predict psychiatric symptoms relatively independently, with little interaction among them.

Table 4 and Figure 2 show the interaction found between EMS and ELS in the regression analysis. The direct effect (c') of ELS $(R=0.23, p<0.001,95 \% \mathrm{Cl} 0.07$ $0.3)$ on psychiatric symptoms was lower than the total effect (c) obtained $(r=0.72, p<0.001,95 \% \mathrm{Cl} 0.5-0.9)$, which confirms the existence of a clear mediation effect between the two variables. In this case, EMS exerted an

Table 4 Mediation model of early maladaptive schemas (predictor variable), harm avoidance (mediator variable), and psychiatric symptoms (output or result variable)

\begin{tabular}{lc}
\hline Effects & $\mathrm{R}$ \\
\hline Total (c) & $0.72^{*}$ \\
Direct (c') & $0.23^{*}$ \\
Indirect (a.b) & $0.49^{*}$ \\
Indirect/total (a.b/c) & $0.68^{*}$
\end{tabular}

$a=$ effect of early life stress on harm avoidance; $b=$ effect of harm avoidande on psychiatric symptoms; $c=$ total effect of early life stress on psychiatric symptoms; $c^{\prime}$ = direct effect of early life stress on psychiatric symptoms.

Bootstrapping $=5,000$ resamples $(95 \%$ confidence interval). $* p<0.001$.

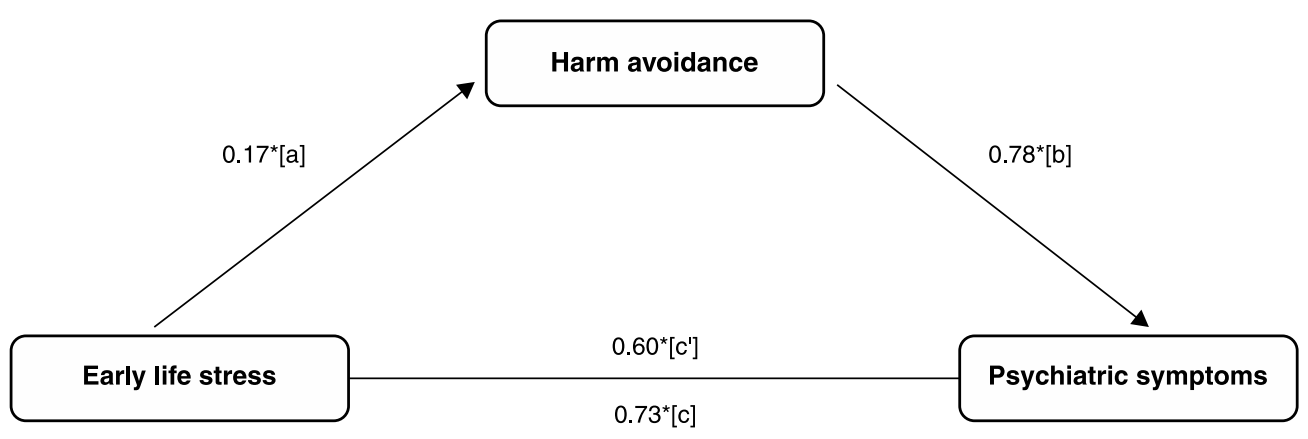

Figure 1 Mediation model of early life stress, harm avoidance, and psychiatric symptoms. a = effect of early life stress on harm avoidance; $b=$ effect of harm avoidande on psychiatric symptoms; $c=$ total effect of early life stress on psychiatric symptoms; $c^{\prime}=$ direct effect of early life stress on psychiatric symptoms. ${ }^{*} p<0.001$. 


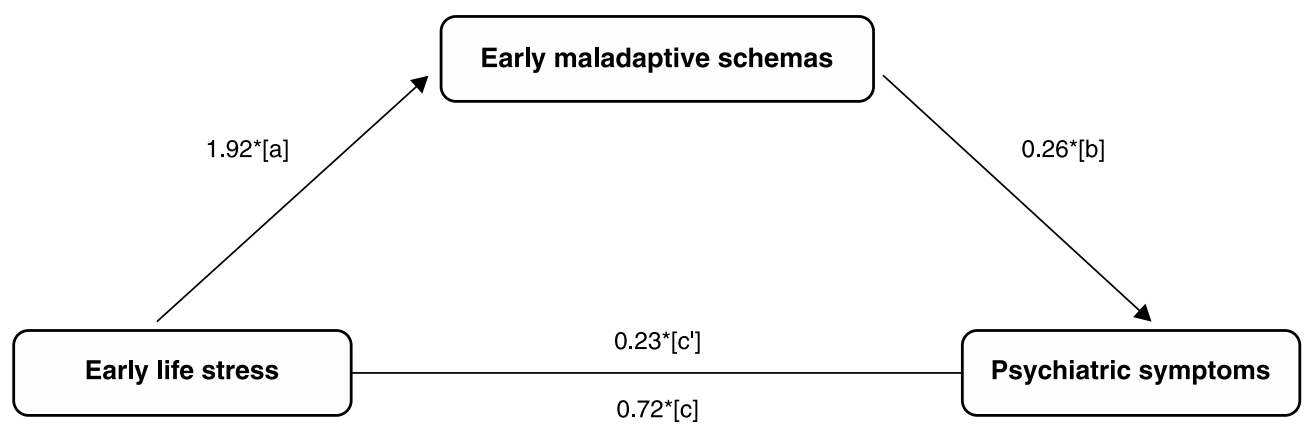

Figure 2 Mediation model of early life stress, early maladaptive schemas, and psychiatric symptoms. a = effect of early life stress on harm avoidance; $b=$ effect of harm avoidande on psychiatric symptoms; $c=$ total effect of early life stress on psychiatric symptoms; $c^{\prime}=$ direct effect of early life stress on psychiatric symptoms. ${ }^{*} p<0.001$.

indirect mediation effect $(\alpha . b)(r=0.49, \mathrm{p}<0.001,95 \% \mathrm{Cl}$ 0.3-0.7) that explained $68 \%$ of the psychiatric symptoms, compared to $32 \%$ explained by ELS. Thus, ELS significantly predicts the emergence of psychiatric symptoms, and when it is associated with EMS the possibility of developing psychiatric symptoms increases substantially.

\section{Discussion}

The main objective of this study was to examine how temperament factors and EMS jointly mediate the relation between ELS and psychiatric symptoms in a non-clinical sample. Important relationships were found between ELS, $\mathrm{HA}$, EMS, and psychiatric symptoms, and these variables predict the development of psychiatric symptoms in adulthood. However, most of the predictive power of ELS for psychiatric symptoms was mediated by HA and EMS. The results demonstrate the importance of these variables for a fuller explanation of the widely reported association between childhood trauma and mental disorders.

Although in this study HA only had a sublte effect on the power of ELS to predict psychiatric symptoms, it has been frequently cited in the literature as a factor in the development of mental disorders. ${ }^{11}$ According to our results, HA had less effect than EMS. Apparently, both variables are independent predictors of psychiatric symptoms, which was expected. One possible reason for this might be that temperament factors such as HA have a predominantly hereditary nature, i.e. they are largely unresponsive to environmental influences and are stable over time. ${ }^{9}$ If such were the case, HA would be little influenced by ELS, although stressful events could have a greater impact on psychiatric symptoms, depending on the way they are interpreted and internalized, as well as the strategies used since the event. ${ }^{23}$

EMS had a significant mediation effect on ELS and psychiatric symptoms, confirming that ELS is one of the most influential factors in the development of EMS, ${ }^{17,24}$ as well as the relationship between EMS and various mental disorders. ${ }^{14}$

Other studies have also found that EMS mediates early stressors and psychiatric symptoms. In a mediation model with insecure attachment, EMS, and various psychiatric symptoms, Roelofs et al. ${ }^{25}$ found that EMS has some effect, mainly through disconnection and rejection, which mediate insecure attachment and emotional problems. Wright et al. ${ }^{26}$ investigated the relationship between abuse, emotional neglect, and psychiatric symptoms in students and observed that vulnerability to harm, embarrassment, and self-sacrifice mediated anxiety and depression symptoms in the sample. Defectiveness/shame and vulnerability to harm also had a mediation effect on dissociation symptoms. ${ }^{24}$

Carr \& Francis investigated the mediation effect of EMS on various types of childhood experiences and symptoms of avoidant personality disorder, finding that subjugation, abandonment, and emotional inhibition mediate the relationship between an overprotective mother and family sociability in childhood and avoidant personality symptoms. ${ }^{18}$ Thimm investigated the mediation effect of EMS on parenting styles and personality disorder symptoms, finding that the disconnection and rejection domain (between maternal rejection and less maternal emotional warmth) effected personality disorder symptoms. ${ }^{27}$

Thus, the EMS-based mediation model identified in this study is supported by several other investigations. The results of the present study confirm the predictive effect of ELS on psychiatric symptoms (regardless of EMS to some degree), which was an expected outcome, since other factors such as genetics, ${ }^{7,28}$ neuroendocrine origin, ${ }^{9}$ and temperament, ${ }^{10}$ also mediate this relationship. However, EMS potentiates the predictor effect of ELS on psychiatric symptoms, and EMS has a significant mediation effect between ELS and psychiatric symptoms.

It can be suggested that the mediating effect of EMS between ELS and psychiatric symptoms occurs due to the influence of ELS on the structuring of EMS. Self-image and self-image in relation to others are formed, for the most part, by episodic events that are stored in a longterm memory system, which gradually takes on structure and schematic functioning. ${ }^{29}$ Self-image begins to exert significant influence on a person's information processing during situations that somehow activate these schemas or are emotionally mobilizing. Traumatic childhood events can influence the formation of EMS, which are a factor in the development of psychiatric symptoms. However, it should be pointed out that EMS can also be formed later and without ELS. However, the EMS formed in such cases tend to be less harmful. ${ }^{12}$ 
Although correlational studies cannot establish causal relations among the variables they investigate, it is proposed that the temperament factor HA, due to its more hereditary nature, could function as an element of vulnerability, which, when combined with ELS, can lead to greater susceptibility for developing psychiatric symptoms. ${ }^{11,16}$ On the other hand, EMS emerge under the direct influence of ELS. Therefore, both HA and EMS are means by which ELS impacts the development of psychiatric symptoms.

The data presented here are in accordance with vulnerability models, which seek to explain the relationship between childhood trauma and an increased risk of mental disorders, including the influence of an individual's characteristics and vulnerabilities. ${ }^{30}$ In the present study, it was determined that ELS, HA, and the development of EMS significantly influence the emergence of psychiatric symptoms, thus indicating vulnerability factors that can help clarify the toxic effects of ELS.

The results of this study provide important evidence about the mediating role that EMS and dysfunctional personality characteristics have on ELS and the development of psychiatric symptoms. Important advances have identified neuroendocrine variables as the most influential mediators in the development of psychiatric symptoms due to ELS. Efforts are currently being made to identify the epigenetic effect of stress in humans. ${ }^{31}$ However, despite the unquestionable importance of this knowledge, it should be pointed out that other types of mediators may have similar relevance, such as EMS and personality factors, which have been analyzed together for the first time in the present study, identifying elements that can be managed or redefined through psychotherapeutic measures. This can lead to effective strategies for emotional regulation in individuals with a history of ELS.

Thus, the results of this study reinforce the wellestablished understanding that early stress can be harmful to the psychological well-being of its victims. The study provides an explanatory model for this relationship that includes other psychological factors that can influence the development of mental disorders, in this case HA and EMS. It is important to point out that early stress in people with mental disorders usually leads to a worse prognosis in psychotherapy and pharmacological treatment ${ }^{32,33}$ than in those without such a history, which highlights the need for further studies on the specific characteristics of this population. A better understanding of the relationship of early stress, personality characteristics and EMS can be useful for developing psychotherapeutic methods that consider the specificities associated with these conditions, increasing the chance of better support for victims, including adequate support in childhood, when prevention is a greater possibility by stimulating protective factors that can minimize the harmful effects of early stress.

In terms of limitations, the first one is regarding the sample, since it mainly consisted of university students and women, which could have led to some bias in the results. Less homogenous samples should be used in further studies. Another important limitation was the lack of investigation into other forms of adversity and abuse, such as a mental disorder or death of one of the parents, divorce, etc. Other types of adversity may have similar influence to the more commonly investigated stressors. Using an instrument to screen for non-diagnosed psychiatric symptoms is also a limitation, since it does not allow more accurate data comparisons with clinically diagnosed populations.

The collection method for early stress data should also be considered a limitation. Memory is subject to bias associated with the encoding and storage of information (which are affected by the emotional content of the event), bias during the reconsolidation the event has undergone until the present moment, and mood congruence bias associated with information retrieval. However, such bias does not completely invalidate the reliability of the information, since it was obtained through retrospective instruments found to have good reliability, even in clinical populations. ${ }^{34}$ The CTQ instrument used in this study also has good test-retest validity. ${ }^{19}$

Another challenge for investigations of early childhood and adolescent stress is determining the period in which the event occurred and whether the resulting harm differs according to age. Some studies have indicated 12 years of age as a remarkable cut-off point for distinguishing the type of harm that can emerge from trauma, based on the fact that the transition to puberty is a critical development period, that involves a greater risk of developing certain mental disorders. ${ }^{35}$ Some authors, in view of brain development factors, believe that early stress at can impact functions associated with specific structures, which are more susceptible at specific times to environmental effects. ${ }^{6,7}$

This study did not investigate the identity of the perpetrator(s) of ELS or recurrent abuse/neglect, which should be considered a limitation. It has been suggested that when the abuser is the mother or the father, the potential effects tend to be more serious and permanent. ${ }^{36}$ Other studies have indicated that in cases of multiple trauma types, there is a corresponding increase in psychiatric symptoms. ${ }^{37,38}$

\section{Acknowledgements}

This study received financial support from Conselho Nacional de Desenvolvimento Científico e Tecnológico (CNPq).

\section{Disclosure}

The authors report no conflicts of interest.

\section{References}

1 Teicher MH, Samson JA. Annual Research Review: Enduring neurobiological effects of childhood abuse and neglect. J Child Psychol Psychiatry. 2016;57:241-66.

2 Carr CP, Martins CM, Stingel AM, Lemgruber VB, Juruena MF. The role of early life stress in adult psychiatric disorders: a systematic review according to childhood trauma subtypes. J Nerv Ment Dis. 2013;201:1007-20.

3 Kalmakis KA, Chandler GE. Adverse childhood experiences: towards a clear conceptual meaning. J Adv Nurs. 2014;70:1489-501. 
4 Hovens JG, Giltay EJ, Wiersma JE, Spinhoven P, Penninx BW, Zitman FG. Impact of childhood life events and trauma on the course of depressive and anxiety disorders. Acta Psychiatr Scand. 2012;126: 198-207.

5 Zhang T, Chow A, Wang LL, Dai YF, Xiao ZP. Role of childhood traumatic experience in personality disorders in China. Compr Psychiatry. 2012;53:829-36.

6 Lupien SJ, McEwen BS, Gunnar MR, Heim C. Effects of stress throughout the lifespan on the brain, behaviour and cognition. Nat Rev Neurosci. 2009;10:434-45.

7 Teicher $\mathrm{MH}$, Samson JA. Childhood maltreatment and psychopathology: a case for ecophenotypic variants as clinically and neurobiologically distinct subtypes. Am J Psychiatry. 2013;170: 1114-33.

8 Reynolds RM. Glucocorticoid excess and the developmental origins of disease: two decades of testing the hypothesis--2012 Curt Richter award winner. Psychoneuroendocrinology. 2013;38:1-11.

9 Cloninger CR, Svrakic DM, Przybeck TR. A psychobiological model of temperament and character. Arch Gen Psychiatry. 1993;50: 975-90.

10 Svrakic DM, Draganic S, Hill K, Bayon C, Przybeck TR, Cloninger CR. Temperament, character, and personality disorders: etiologic, diagnostic, treatment issues. Acta Psychiatr Scand. 2002;106:189-95.

11 Fassino S, Amianto F, Sobrero C, Abbate Daga G. Does it exist a personality core of mental illness? A systematic review on core psychobiological personality traits in mental disorders. Panminerva Med. 2013;55:397-413.

12 Young JE, Klosko JS, Weishaar ME. Schema therapy: a practitioner's guide. New York: Guilford; 2006.

13 Tezel FK, Kişlak ST, Boysan M. Relationships between childhood traumatic experiences, early maladaptive schemas and interpersonal styles. Noro Psikiyatr Ars. 2015;52:226-32.

14 Shorey RC, Elmquist J, Anderson S, Stuart GL. The relationship between early maladaptive schemas, depression, and generalized anxiety among adults seeking residential treatment for substance use disorders. J Psychoactive Drugs. 2015;47:230-8.

15 Carver CS, Johnson SL, McCullough ME, Forster DE, Joormann J. Adulthood personality correlates of childhood adversity. Front Psychol. 2014;5:1357

16 de Carvalho HW, Pereira R, Frozi J, Bisol LW, Ottoni GL, Lara DR. Childhood trauma is associated with maladaptive personality traits. Child Abuse Negl. 2014;44:18-25.

17 Roemmele. M, Messman-Moore TL. Child abuse, early maladaptive schemas, and risky sexual behavior in college women. J Child Sex Abus. 2011;20:264-83.

18 Carr SN, Francis AJ. Do early maladaptive schemas mediate the relationship between childhood experiences and avoidant personality disorder features? A preliminary investigation in a non-clinical sample. Cognit Ther Res. 2010;34:343-58.

19 Bernstein DP, Stein JA, Newcomb MD, Walker E, Pogge D, Ahluvalia $\mathrm{T}$, et al. Development and validation of a brief screening version of the Childhood Trauma Questionnaire. Child Abuse Negl. 2003;27: 169-90.

20 Gadow KD, Sprafkin J, Weiss M. Adult self-report inventory-4 manual Stony Brook: Checkmate Plus; 2004.

21 Hayes AF. Process: A versatile computational tool for observed variable mediation, moderation, and conditional process modeling
[Internet]. 2012. [cited 2015 Aug 17]. www.afhayes.com/public/ process2012.pdf

22 Hayes AF, Scharkow M. The relative trustworthiness of inferential tests of the indirect effect in statistical mediation analysis does method really matter? Psychol Sci. 2013;24:1918-27.

23 Stenbæk DS, Jensen CG, Holst KK, Mortensen EL, Knudsen GM, Frokjaer VG. Does harm avoidance mediate effects of recollected parental bonding on mental distress in adulthood? Compr Psychiatry. 2014;55:1007-14.

24 Calvete E. Emotional abuse as a predictor of early maladaptive schemas in adolescents: contributions to the development of depressive and social anxiety symptoms. Child Abuse Negl. 2014;38: 735-46.

25 Roelofs J, Onckels L, Muris P. Attachment quality and psychopathological symptoms in clinically referred adolescents: the mediating role of early maladaptive schema. J Child Fam Stud. 2013;2: 377-85.

26 Wright MO, Crawford E, Del Castillo D. Childhood emotional maltreatment and later psychological distress among college students: the mediating role of maladaptive schemas. Child Abuse Negl. 2009;33:59-68.

27 Thimm JC. Mediation of early maladaptive schemas between perceptions of parental rearing style and personality disorder symptoms. J Behav Ther Exp Psychiatry. 2010;41:52-9.

28 Gershon A, Sudheimer K, Tirouvanziam R, Williams LM, O'Hara R. The long-term impact of early adversity on late-life psychiatric disorders. Curr Psychiatry Rep. 2013;15:352.

29 Conway MA. Memory and the self. J Mem Lang. 2005;53:594-628.

30 Nugent NR, Tyrka AR, Carpenter LL, Price LH. Gene-environment interactions: early life stress and risk for depressive and anxiety disorders. Psychopharmacology (Berl). 2011;214:175-96.

31 Yehuda R, Daskalakis NP, Bierer LM, Heather NB, Klengel T, Holsboer $\mathrm{F}$, Binder EB. Holocaust exposure induced intergenerational effects on FKBP5 methylation. Biol Psychiatry. 2016;80:372-80.

32 Nemeroff CB, Heim CM, Thase ME, Klein DN, Rush AJ, Schatzberg $A F$, et al. Differential responses to psychotherapy versus pharmacotherapy in patients with chronic forms of major depression and childhood trauma. Proc Natl Acad Sci U S A. 2003;100:14293-6.

33 Williams LM, Debattista C, Duchemin AM, Schatzberg AF, Nemeroff CB. Childhood trauma predicts antidepressant response in adults with major depression: data from the randomized international study to predict optimized treatment for depression. Transl Psychiatry. 2016;6:e799.

34 Hardt J, Rutter M. Validity of adult retrospective reports of adverse childhood experiences: review of the evidence. J Child Psychol Psychiatry. 2004;45:260-73.

35 Grover KE, Carpenter LL, Price LH, Gagne GG, Mello AF, Mello MF, et al. The relationship between childhood abuse and adult personality disorder symptoms. J Pers Disord. 2007;21:442-7.

36 McCarthy MC, Lumley MN. Sources of emotional maltreatment and the differential development of unconditional and conditional schemas. Cogn Behav Ther. 2012;41:288-97.

37 Arata CM, Langhinrichsen-Rohling J, Bowers D, O’Brien N. Differential correlates of multi-type maltreatment among urban youth. Child Abuse Negl. 2007;31:393-415.

38 Finkelhor D, Ormrod RK, Turner HA. Poly-victimization: a neglected component in child victimization. Child Abuse Negl. 2007;31:7-26. 